\title{
Prevalence of salivary gland hypertrophy syndrome in laboratory colonies and wild flies of Glossina pallidipes in Ethiopia
}

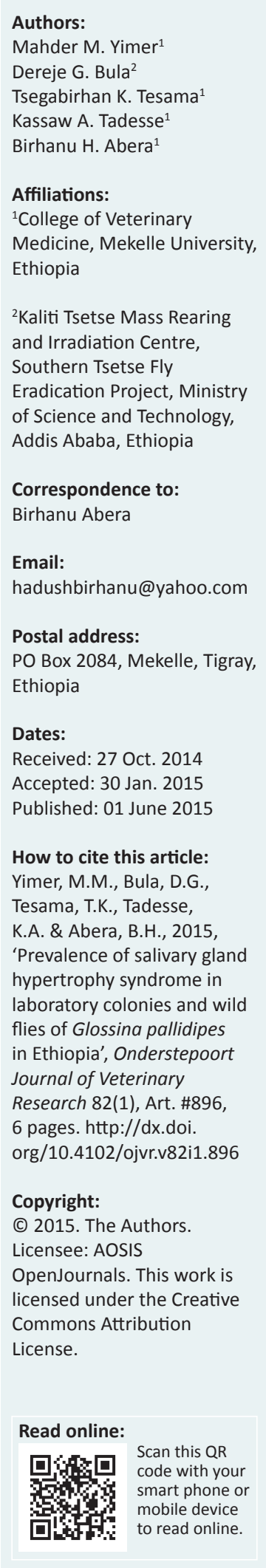

Glossina pallidipes salivary gland hyperplasia (GpSGH) syndrome caused by the salivary gland hyperplasia virus reduces the reproduction potential of tsetse flies, posing a serious threat for rearing of sufficient colonies for use of tsetse and trypanosome control using the sterile insect technique. This research was conducted in the Kaliti Tsetse Mass Rearing and Irradiation Centre in Ethiopia with the objective of studying the prevalence of GpSGH syndrome in laboratory colonies of G. pallidipes (Tororo and Arbaminch) reared for release in the implementation of the sterile insect technique and a field strain of G. pallidipes Arbaminch. Presence or absence of GpSGH was determined when pathological features of the salivary gland were revealed after dissection. The overall prevalence of GpSGH syndrome in laboratory colonies was 48.3\% $(747 / 1548)$ with a statistically significant $(z=17.30, p=0.001)$ prevalence of $70.2 \%(544 / 775)$ in Arbaminch colonies and 26.26\% (203/773) in Tororo colonies. The prevalence of GpSGH in laboratory flies fed according to the clean blood feeding protocol was $68.9 \%$ and $22.4 \%$ in Arbaminch and Tororo strains respectively. It was $70.5 \%$ and $27.2 \%$ respectively in laboratory colonies of Arbaminch and Tororo strains fed according to the standard membrane feeding protocol. The difference in prevalence of the disease between the two feeding protocols was not statistically significant in either Arbaminch $(z=0.361, p=0.359)$ or Tororo $(z=1.22, p=0.111)$ strains. The prevalence of SGH in wild G. pallidipes Arbaminch strain was 3\% (15/500) and was significantly $(z=23.61, p<0.001)$ lower than in the laboratory strain. The effect of age and density-related stress on the development of GpSGH was not statistically significant. The prevalence of GpSGH in the newly emerging (teneral) flies in the laboratory colonies was $66.7 \%$ and $20 \%$ in the Arbaminch and Tororo strains respectively. For all considered risk factors, the prevalence was much higher in G. pallidipes Arbaminch laboratory colonies.

\section{Introduction}

The debilitating diseases African sleeping sickness in humans, caused by Trypanosoma brucei gambiense and T. b. rhodesiense and African animal trypanosomosis (AAT), caused by T. b. brucei, T. congolense and T. vivax, are cyclically transmitted by tsetse flies (Glossina species) (Simarro, Louis \& Jannin 2003; World Health Organization 2003). In Ethiopia, an area of about $220000 \mathrm{~km}^{2}$ is infested by G. morsitans submorsitans, G. pallidipes, G. tachinoides, G. fuscipes fuscipes and G. longipennis, contributing as biological vectors of T. congolense, T. vivax and T. brucei (Abebe 2005). Tesfaye et al. (2012) reported that trypanosomosis is responsible for substantial economic losses through cattle mortality, cost incurred for drug purchase and other control interventions and loss of draft power of infected oxen. On the other hand, Shaw et al. (2014) indicated huge economic advantages that can be accessed from implementing tsetse and trypanosomosis control interventions in currently infected areas.

Control of tsetse flies and use of trypanocidal drugs for treatment or prophylaxis are the two control options for trypanosomosis; however, both have many drawbacks, such as the high cost of purchase, environmental pollution by insecticides, increasing trypanocidal drug resistance and lack of new drugs to replace them (Chaka \& Abebe 2003). Vector control through the sterile insect technique (SIT) is an alternative strategy for the sustainable management of these diseases (Leak 1998). Successful eradication of G. austeni on the island of Unguja, Republic of Tanzania, entailed an area-wide integrated pest management approach (Hendrichs et al. 2007) that included the release of sterile male flies (Vreysen et al. 2000), and serves as a starting point for the wider recommendation of the technique in Africa. In 1996, the government of Ethiopia, in collaboration with the United Nations' International Atomic Energy Agency (IAEA), initiated a tsetse eradication project called the Southern Rift Valley Tsetse Eradication Project (STEP) using SIT (Alemu et al. 2007; Feldmann et al. 2005). 
SIT requires production of surplus males that are later gamma sterilised for field release and maintenance of females breeding for colony turnover. Failure to raise big colonies as a result of the presence of G. pallidipes salivary gland hypertrophy/hyperplasia virus (GpSGHV) is the main stumbling block for SIT application and could detrimentally affect tsetse control, with consequent serious health and economic implications (Abd-Alla et al. 2010; Sasanya et al. 2010). The virus replicates in the nucleus of salivary gland cells, causing distinct tissue hypertrophy in the female milk gland and male gonadal tissues, resulting in ovarian abnormalities and testicular degeneration, thus reducing the reproductive potential in both sexes of G. pallidipes (Sang et al. 1999). Vertical transmission of GpSGHV from mother to offspring either by trans-ovarian transfer of virus to embryo (Jura, Otieno \& Chimtawi 1989) or via virus-infected milk glands to developing larvae (Sang et al. 1999) are the principal transmission routes. In laboratory-maintained flies, horizontal transmission during in vitro feeding of blood provided under a silicone membrane (Feldmann 1994) was believed to be a major route of virus infection, which is enhanced as a result of confinement in rearing cages (Malele et al. 2013).

The presence of G. pallidipes salivary gland hypertrophy/ hyperplasia $(\mathrm{GpSGH})$ in colonies at Kaliti Tsetse Mass Rearing and Irradiation Centre (TMRIC) and Arbaminch wild colonies was reported by Abd-Alla et al. (2010), constituting a potential challenge to maintaining sufficient laboratoryreared tsetse fly colonies to be irradiated and released. Determining the magnitude and associated risk factors of the disease could contribute to designing applicable control options for the virus infection in tsetse flies. Therefore, this study was designed to determine the prevalence of GpSGH syndrome in two laboratory colonies of G. pallidipes (Tororo and Arbaminch) and a wild strain of G. pallidipes Arbaminch.

\section{Materials and methods Description of the study areas}

This study was conducted from November 2011 to June 2012. The laboratory work was conducted in the TMRIC, Kaliti, Addis Ababa, located $8^{\circ} 50^{\prime} \mathrm{N}$ to $9^{\circ} 06^{\prime} \mathrm{N}$ and $38^{\circ} 05^{\prime} \mathrm{E}$ to $39^{\circ} 05^{\prime} \mathrm{E}$ (Addis Ababa City Administration, 1998, cited by Nigatu, Rajan \& Bizunesh 2011). Wild G. pallidipes Arbaminch strains were collected at selected sampling points in the district of Arbaminch in the Gamo Goffa Zone of the Southern Nations, Nationalities, and Peoples' Region (SNNPR), about 500 km south of Addis Ababa, located between $4^{\circ} 45^{\prime} \mathrm{N}$ and $7^{\circ} 15^{\prime} \mathrm{N}$ and $36^{\circ} 40^{\prime} \mathrm{E}$ and $35^{\circ} 20^{\prime} \mathrm{E}$. The climate varies between humid and windy, with annual precipitation between $300 \mathrm{~mm}$ and $700 \mathrm{~mm}$ in the lowlands and between $500 \mathrm{~mm}$ and $1200 \mathrm{~mm}$ in the highlands. The average temperature ranges between $11^{\circ} \mathrm{C}$ and $38^{\circ} \mathrm{C}$ (Alemu et al. 2007).

\section{Tsetse fly populations}

Tsetse flies from two laboratory colonies of G. pallidipes were used in this study, (1) a colony maintained and originating from pupae collected near Tororo, Uganda (Tororo colony) and (2) a colony established at the TMRIC from pupae collected near Arbaminch (Arbaminch colony) since 1996. To check the condition of GpSGH syndrome in the field populations, wild G. pallidipes of the Arbaminch strain were collected from the Arbaminch area. Collection and sex identification based on hypopygium structure were carried out as described previously (FAO 1992). The flies were handled in standard cages and transported to the TMRIC. These flies were dissected immediately on arrival; no feeding and mixing with laboratory colonies was allowed. Age was not determined in these flies.

\section{Blood feeding protocols}

Two protocols for feeding the flies were used, (1) the standard membrane feeding protocol, whereby successive cages with flies were offered a blood meal on the same membrane (Feldmann 1994) and (2) a clean blood feeding protocol, whereby each cage of flies was provided with new, sterile defibrinated bovine blood at each meal, which prevents the flies from picking up the virus from the blood used for feeding previous cages (Abd-Alla et al. 2010). Both feeding systems were evaluated for their effect on the prevalence of GpSGH. The clean blood feeding protocol was conducted in seven cages of each laboratory strain, containing 30 male flies per cage, at each feeding time. These flies were progeny of parent stocks that were fed under the standard membrane feeding protocol. In the clean feeding protocol, newly-emerged (teneral) G. pallidipes flies of both strains were offered a clean blood meal and thereafter were always fed on fresh, clean blood during the entire period of the experiment (Table 1). The tsetse colonies used to study the effect of density-related stress (Table 2) and age (Table 3) were fed using the standard membrane feeding protocol.

\section{Effect of stress (density) on the prevalence of GpSGH syndrome}

Male teneral flies were selected randomly from both strains of the G. pallidipes colonies and maintained in standard colony holding cages $(20 \mathrm{~cm} \times 20 \mathrm{~cm} \times 5 \mathrm{~cm})$ at densities of 38,68 and 120 flies per cage (Table 2), as indicated by Abd-Alla et al. (2010). Each cage was maintained at $24{ }^{\circ} \mathrm{C}$ and $70 \%-80 \%$ humidity for 20 days. After 20 days, all the tsetse flies were dissected and the condition of the salivary glands was recorded.

TABLE 1: Prevalence of Glossina pallidipes salivary gland hyperplasia in Glossina pallidipes Arbaminch and Glossina pallidipes Tororo laboratory colonies (clean membrane feeding).

\begin{tabular}{lccccccc}
\hline Feeding time & \multicolumn{3}{c}{ Glossina pallidipes Arbaminch } & & \multicolumn{3}{c}{ Glossina pallidipes Tororo } \\
\cline { 2 - 4 } & Dissected & Positive & $\mathbf{\%}$ & & Dissected & Positive & \% \\
\hline Teneral (unfed) & 30 & 20 & 66.7 & & 30 & 6 & 20.0 \\
Week 3 & 28 & 19 & 67.9 & & 28 & 6 & 21.4 \\
Week 6 & 29 & 20 & 69.0 & & 27 & 6 & 22.2 \\
Week 9 & 26 & 18 & 69.2 & & 30 & 7 & 23.3 \\
Week 12 & 27 & 19 & 70.4 & & 21 & 5 & 23.8 \\
Week 15 & 21 & 15 & 71.4 & & 16 & 4 & 25.0 \\
\hline Total & $\mathbf{1 6 1}$ & $\mathbf{1 1 1}$ & $\mathbf{6 8 . 9}$ & & $\mathbf{1 5 2}$ & $\mathbf{3 4}$ & $\mathbf{2 2 . 4}$ \\
\hline
\end{tabular}


TABLE 2: Effect of stress (density) on prevalence of Glossina pallidipes salivary gland hyperplasia in Glossina pallidipes Arbaminch and Glossina pallidipes Tororo laboratory colonies (standard membrane feeding).

\begin{tabular}{|c|c|c|c|c|c|c|c|c|c|c|c|}
\hline \multirow[t]{2}{*}{ Density } & \multicolumn{5}{|c|}{ Glossina pallidipes Arbaminch } & \multicolumn{6}{|c|}{ Glossina pallidipes Tororo } \\
\hline & Dissected & Positive & $\%$ & Odds ratio & $p$-value & Density & Dissected & Positive & $\%$ & Odds ratio & $p$-value \\
\hline 38 & 32 & 22 & 68.8 & - & - & 38 & 31 & 7 & 22.6 & - & - \\
\hline 68 & 55 & 38 & 69.1 & 1.17 & 0.739 & 68 & 50 & 13 & 26.0 & 1.20 & 0.729 \\
\hline 120 & 92 & 64 & 69.6 & 1.20 & 0.679 & 120 & 80 & 21 & 26.3 & 1.22 & 0.690 \\
\hline 226 & 179 & 124 & 69.3 & - & - & - & 161 & 41 & 21.5 & - & - \\
\hline
\end{tabular}

TABLE 3: Effect of age on the prevalence of Glossina pallidipes salivary gland hyperplasia in Glossina pallidipes Arbaminch and Glossina pallidipes Tororo laboratory colonies (standard membrane feeding).

\begin{tabular}{|c|c|c|c|c|c|c|c|c|c|c|}
\hline \multirow[t]{2}{*}{ Age } & \multicolumn{5}{|c|}{ Glossina pallidipes Arbaminch colony } & \multicolumn{5}{|c|}{ Glossina pallidipes Tororo colony } \\
\hline & Dissected & Positive & $\%$ & Odds ratio & $p$-value & Dissected & Positive & $\%$ & Odds ratio & $p$-value \\
\hline Day 10 & 88 & 60 & 68.2 & - & - & 89 & 21 & 23.5 & - & - \\
\hline Week 2 & 29 & 20 & 69.0 & 1.04 & 0.937 & 25 & 6 & 24.0 & 1.02 & 0.966 \\
\hline Week 3 & 17 & 12 & 70.6 & 1.12 & 0.845 & 30 & 8 & 26.7 & 1.18 & 0.735 \\
\hline Week 4 & 20 & 14 & 70.0 & 1.09 & 0.874 & 26 & 7 & 26.9 & 1.36 & 0.527 \\
\hline Week 5 & 27 & 19 & 70.4 & 1.10 & 0.830 & 29 & 8 & 27.6 & 1.23 & 0.665 \\
\hline Week 6 & 24 & 17 & 70.8 & 1.13 & 0.804 & 25 & 7 & 28.0 & 1.26 & 0.652 \\
\hline Week 7 & 28 & 20 & 71.4 & 1.17 & 0.746 & 27 & 8 & 29.6 & 1.36 & 0.527 \\
\hline Week 8 & 24 & 17 & 70.8 & 1.13 & 0.804 & 30 & 9 & 30.0 & 1.39 & 0.486 \\
\hline Week 9 & 25 & 18 & 72.0 & 1.20 & 0.716 & 28 & 8 & 28.6 & 1.30 & 0.595 \\
\hline Week 10 & 29 & 21 & 72.4 & 1.23 & 0.669 & 24 & 7 & 29.2 & 1.33 & 0.576 \\
\hline Week 11 & 22 & 16 & 72.7 & 1.24 & 0.680 & 17 & 5 & 29.4 & 1.35 & 0.610 \\
\hline Week 12 & 26 & 19 & 73.1 & 1.27 & 0.635 & 26 & 8 & 30.8 & 1.44 & 0.460 \\
\hline Week 13 & 15 & 11 & 73.3 & 1.28 & 0.691 & 23 & 7 & 30.4 & 1.42 & 0.501 \\
\hline Week 15 & 23 & 17 & 73.9 & 1.32 & 0.596 & 16 & 5 & 31.3 & 1.47 & 0.515 \\
\hline Week 16 & 19 & 14 & 73.7 & 1.3 & 0.638 & 19 & 6 & 31.6 & 1.49 & 0.468 \\
\hline Total & 435 & 309 & 71.0 & - & - & 460 & 128 & 27.8 & - & - \\
\hline
\end{tabular}

\section{Effect of age on the prevalence of GpSGH syndrome}

Tsetse flies in the Kaliti TMRIC live for a maximum period of 17 weeks (4 months). A total of 17 cages of different age groups of both strains were selected, each containing 30 male flies kept on a regimen of clean membrane blood feeding three times per week. Age was determined from standard records available in the centre. From each age group, a minimum of 15 tsetse flies of both strains (one cage of each) were dissected at the age of 10 days and at weekly intervals from week 2-16 (Table 3).

\section{Tsetse fly dissection procedures and identification of post-mortem GpSGHV lesions}

Tsetse flies were dissected under a stereo microscope according to the procedures indicated by the FAO (1992). Immediately afterwards the glands were mounted in $5 \%$ saline and examined under the microscope. Pathological features of salivary gland hypertrophy/hyperplasia virus (SGHV) described by Gouteux (1987) and Verena et al. (2011) were used to distinguish between infected and non-infected flies.

Briefly, on dissection of the tsetse flies, normal salivary glands were transparent and the diameter of the distal part of the gland was about $100 \mu \mathrm{m}$, with almost no variation throughout the life of the tsetse fly, whilst hypertrophied glands were enlarged (diameter could reach up to $1.1 \mathrm{~mm}$ more than their normal thickness) and chalky-white or bluish in appearance.

\section{Statistical analysis}

Statistical Package for Social Sciences (SPSS) version 17 statistical software (SPSS 2007) was used for data entry and analysis. Comparison of the prevalence (\%) of GpSGH was made by logistic regression. Odds ratio was used to report the effect of the discrete study variables (stress and age) on the development of GpSGH syndrome. The Z-test for comparison of two population proportions was used to check the statistical significance of the prevalence of GpSGH in the strains, prevalence related to standard membrane and clean blood feeding protocols of the laboratory colonies and wild tsetse fly strains from the Arbaminch area. In all analyses, the confidence level was held at 95\% (Thrusfield 2005).

\section{Results}

A total of 2048 tsetse flies (1548 laboratory adapted and 500 wild) were dissected. The overall prevalence of GpSGH syndrome in laboratory colonies was $48.3 \%(747 / 1548)$ with a statistically significant $(z=17.30, p<0.001)$ prevalence of $70.2 \%(544 / 775)$ in Arbaminch and $26.3 \%(203 / 773)$ in Tororo colonies (Tables 1-3). The prevalence of GpSGH in laboratory flies fed using the clean blood feeding protocol was $68.9 \%(111 / 161)$ and $22.4 \%(34 / 152)$ in Arbaminch and Tororo strains respectively (Table 1). Likewise, the prevalence in laboratory colonies fed using the standard membrane feeding protocol was $70.5 \%(433 / 614)$ and $27.2 \%$ $(169 / 621)$ in Arbaminch and Tororo strains respectively 
(Tables $2 \& 3$ ). This variation in prevalence of the disease with both feeding protocols was not statistically significant in either Arbaminch $(z=0.361, p=0.359)$ or Tororo $(z=1.22$, $p=0.111)$ strains. The prevalence of GpSGH in the wild G. pallidipes Arbaminch strain was 3\% (15/500). A statistically non-significant $(z=1.31, p=0.095)$ difference in prevalence of $2.5 \%(10 / 400)$ and $5 \%(5 / 100)$ in female and male tsetse flies respectively was observed. The prevalence of GpSGH in the G. pallidipes Arbaminch laboratory colony (70.2\%) was significantly higher than in the wild G. pallidipes Arbaminch strain $(3 \%)(z=23.61, p<0.001)$.

In flies fed using the standard membrane feeding protocol, there was a statistically non-significant increase in prevalence of GpSGH syndrome from $68.2 \%$ to $73.7 \%$ in Arbaminch laboratory colonies and from $23.7 \%$ to $31.6 \%$ in Tororo colonies as the age of tsetse flies increased (Table 3). Moreover, as the feeding time on the clean membrane increased, there was a statistically non-significant increase in the prevalence of GpSGH syndrome from $66.7 \%$ to $71.4 \%$ in Arbaminch colonies and from $20.0 \%$ to $25.0 \%$ in Tororo colonies. The prevalence of GpSGH in the newly emerging (teneral) flies was $66.7 \%$ and $20 \%$ in Arbaminch and Tororo strains respectively (Table 1).

As the density of tsetse flies within a cage increased, there was no difference in the prevalence of GpSGH syndrome in either G. pallidipes Arbaminch laboratory colonies or G. pallidipes Tororo colonies (Table 2).

\section{Discussion}

Monitoring the prevalence of GpSGH through dissections is an important component of quality control (Abd-Alla et al. 2010; Abd-Alla et al. 2013a). The prevalence of GpSGH in Arbaminch laboratory colonies fed using standard membrane feeding was $70.5 \%$, which was comparable to results of Abd-Alla et al. (2013b), who indicated a prevalence of $73.84 \%$ on dissection of old Kaliti Arbaminch colonies. The prevalence of GpSGH in Tororo colonies fed using the standard membrane feeding was $27.2 \%$. Abd-Alla et al. (2010) had reported on the prevalence of GpSGH from 2007-2009 in Arbaminch (22.4\%, 43.5\%, 27\% respectively) and Tororo $(\leq 10 \%)$ colonies in the Kaliti TMRIC. The clean feeding regimen decreased the virus copy number (Abd-Alla et al. 2010; Lietze et al. 2009) and is the recommended feeding strategy to reduce the virus load and thus the prevalence of SGH syndrome, according to Abd-Alla et al. (2013b). However, even though some of the tsetse flies dealt within our study (Table 1) were fed using this feeding regimen, the prevalence of the disease was nevertheless high and not statistically different from the prevalence in the flies fed using the standard membrane. The prevalence of GpSGH in Arbaminch strains was significantly higher $(p<0.001)$ than in Tororo strains.

The high prevalence of GpSGH syndrome in the teneral and adult laboratory colonies fed using the clean membrane protocol (Table 1) and the notable proportion in the wild strains could be explained by an infection acquired from the parent stocks. Vertical transmission of SGHV from mother to offspring either by trans-ovarian transfer of virus to embryo (Jura et al. 1989) or via virus-infected milk glands to developing larvae (Sang et al. 1999) has been documented as the principal transmission route. However, the role of horizontal transmission in maintaining and disseminating the infection should not be underestimated, as the virus is continuously shed in the saliva of infected flies (Abd-Alla et al. 2007). The feeding habit of tsetse flies - emptying saliva content prior to feeding into the medium (blood meal) causes virus particle accumulation at each bite, which facilitates the exposure of the healthy susceptible colony (Abd-Alla et al. 2011). Abd-Alla et al. (2010, 2013a) reported that attempts to use the membrane feeding technique to upscale production in a G. pallidipes facility in Ethiopia was extremely challenging because of rapid spread of GPSGHV in the colonies. The absence of a statistically insignificant increase in the prevalence of SGH in G. pallidipes Arbaminch and G. pallidipes Tororo laboratory colonies (Table 2) as the density increased was in agreement with Abd-Alla et al. (2010). Moreover, as the age of the flies increased (Table 3), a statistically non-significant increase in the prevalence of SGH was observed. This could indicate the absence of immunebased resistance in teneral and adult flies and stress factors such as density and age might not play a significant role in disease transmission dynamics.

For all the study variables considered, the prevalence of GpSGH syndrome in G. pallidipes Tororo strain was significantly lower than in G. pallidipes Arbaminch strain. This observation was in agreement with Abd-Alla et al. (2011, 2013a). According to Abd-Alla et al. (2010), this low prevalence in Tororo strains could be as a result of (1) lower pathogenicity of this virus compared to that of the Ethiopian strain, (2) an acquired resistance of the flies in this colony to the virus or (3) a different virus copy number in asymptomatic and symptomatic flies, or a combination of the three.

The prevalence of GpSGH in the wild Arbaminch flies in our study $(3 \%)$ was comparable to the reports of AbdAlla et al. (2013b) in the new Arbaminch laboratory colony $(4.47 \%)$. However, this figure should not be underestimated. According to Kariithi et al. (2013) and Abd-Alla et al. (2007), an asymptomatic course of the virus infection might have contributed to the low prevalence of GpSGH in wild tsetse flies. Using molecular diagnostic tools, Kariithi et al. (2013) reported a prevalence of $68.9 \%$ in Arbaminch wild tsetse populations, in which mother-to-offspring transmission, either trans-ovum or through infected milk glands, is thought to be the most likely mode of transmission of the virus (Jura et al. 1989; Sang et al. 1998).

Successful implementation of an area-wide integrated pest management programme with SIT requires largescale production and release of sterile males of acceptable quality, that is, males capable of competing with wild males for mating with wild tsetse females (Hendrichs et al. 2007). 
However, infections with SGHV markedly reduce the already low natural reproductive potential of tsetse flies kept for breeding purposes. Infected female flies have reduced reproductive organs and male G. pallidipes positive to SGHV infection are unable to inseminate female flies; releasing those infected sterile flies will reduce the efficiency of a sterile male release programme (Gouteux 1987; Mutika et al. 2012). To alleviate the heavy economic losses associated with GpSGHV and ensure successful SIT programmes, various control and prevention strategies are envisaged. Prior to incorporating them in the breeding colonies, parent colonies have to be screened with appropriate diagnostic tools, and a combination of antiviral drug treatment and using the clean feeding system could aid better health management of the breeding colonies (Abd-Alla et al. 2011, 2014).

In conclusion, high infection rates in these colonies warrant replacing the existing breeding colonies with new, healthy ones. Because of the presence of the infection in wild colonies, the selection of new breeding colonies from wild populations has to be based on careful screening with appropriate diagnostic tools. Improved health management strategies coupled with other husbandry practices could contribute to better management of new breeding colonies. Further studies to identify comparative roles of vertical and horizontal transmission cycles on the disease dynamics could help in designing alternative control strategies.

\section{Acknowledgements}

All utilised logistics and finances were received from Kaliti Tsetse Mass Rearing and Irradiation Centre. Moreover, staff members of this institution and the STEP in Arbaminch are acknowledged for their technical support and overall facilitation of the work. The Mekelle University is acknowledged for extending additional support and partially covering the first author's travel costs.

\section{Competing interests}

The authors declare that they have no financial or personal relationships which may have inappropriately influenced them in writing this article.

\section{Authors' contributions}

M.M.Y. (Mekelle University), D.G.B. (Kaliti Tsetse Mass Rearing and Irradiation Centre) and B.H.A. (Mekelle University) designed the research. M.M.Y. and D.G.B. were responsible for collection and analysis of all laboratory and field data. Statistical analysis was made by K.A.T. (Mekelle University). B.H.A., M.M.Y. and T.K.T. (Mekelle University) wrote the article. All authors have contributed to the preparation of the article and approved it for publication.

\section{References}

Abd-Alla, A.M.M., Bergoin, M., Parker, A.G., Maniania, N.K., Vlak, J.M., Bourtzis, K. et al., 2013a, 'Improving sterile insect technique (SIT) for tsetse flies through research on their symbionts and pathogens', Journal of Invertebrate Pathology 112 Suppl., S2-S10. http://dx.doi.org/10.1016/j.jip.2012.07.009
Abd-Alla, A.M.M., Bossin, H., Cousserans, F., Parker, A., Bergoin, M. \& Robinson, A., 2007, 'Development of a non-destructive PCR method for detection of the salivary gland hypertrophy virus (SGHV) in tsetse flies', Journal of Virological Methods 139(2), 143-149. http://dx.doi.org/10.1016/j.jviromet.2006.09.018

Abd-Alla, A.M.M., Kariithi, H.M, Andrew, G.P., Robinson, A.S., Kiflom, M., Bergoin, M. et al., 2010, 'Dynamics of the salivary gland hypertrophy virus in laboratory colonies of Glossina pallidipes (Diptera: Glossinidae)', Viruses 150, 103-110. http://dx.doi.org/10.1016/j.virusres.2010.03.001

Abd-Alla, A.M.M., Kariithi, H.M., Mohamed, A.H., Lapiz, E., Parker, A.G. \& Vreysen, M.J.B., 2013b, 'Managing hytrosavirus infections in Glossina pallidipes colonies: Feeding regime affects the prevalence of salivary gland hypertrophy syndrome', PLoS ONE 8(5), e61875. http://dx.doi.org/10.1371/journal.pone.0061875

Abd-Alla, A.M.M., Marin, C., Parker, A.G. \& Vreysen, M.J.B., 2014, 'Antiviral drug valacyclovir treatment combined with a clean feeding system enhances the suppression of salivary gland hypertrophy in laboratory colonies of Glossina pallidipes' Parasites and Vectors 7(214), 1-4. http://dx.doi.org/10.1186/1756-3305-7-214

Abd-Alla, A.M.M., Salem, T.Z., Parke, A.G., Wang, Y., Jehle, J.A., Vreysen, M.J.B. et al., 2011, 'Universal primers for rapid detection of hytrosaviruses', Journal of Virological Methods 171, 280-283. http://dx.doi.org/10.1016/j.jviromet.2010.09.025

Abebe, G., 2005, 'Trypanosomosis in Ethiopia', Ethiopian Journal of Biological Sciences 4(1), 75-121.

Alemu, T., Kapitano, B., Mekonnen, S., Aboset, G. \& Kiflom, M., 2007, 'Area wide control of tsetse and trypanosomosis: Ethiopian experience in the Southern Rift Valley', in M.J.B Vreysen, A.S. Robinson \& J. Hendrichs (eds.), Area-wide control Valley', in M.J.B Vreysen, A.S. Robinson \& J Hendrichs (eds.), Area-wide control
of insect pests: From research to field implementation, pp. 325-335, Springer, of insect pests: From research to field implementation, pp.
Dordrecht. http://dx.doi.org/10.1007/978-1-4020-6059-5_30

Chaka, H. \& Abebe, G., 2003, 'Drug resistant trypanosomes: A threat to cattle production in the south west of Ethiopia', Revue d'Elevage et de Médecine production in the south west of 'Ethiopia',
Véterinaire des Pays Tropicaux 56(1-2), 33-36.

FAO, 1992, Training manual for tsetse control personnel, Vol. 1, viewed 15 March 2015, from http://www.fao.org/docrep/009/p5178e/P5178E00.htm\#TOC

Feldmann, U., 1994, 'Guidelines for the rearing of tsetse flies using the membrane feeding technique', in J.P.R. Ochieng'-Odero (ed.), Techniques of insect rearing for the development of integrated pest and vector management strategies, pp. 449-471, ICIPE Science Press, Nairobi.

Feldmann, U., Dyck, V.A., Mattioli, R.C. \& Jannin, J., 2005, 'Potential impact of tsetse fly control involving the sterile insect technique', in V.A. Dyck, J. Hendrichs \& A.S. Robinson (eds.), Sterile insect technique: Principles and practice in area-wide integrated pest management, pp. 701-723, Springer, Dordrecht. http://dx.doi. org/10.1007/1-4020-4051-2_27

Gouteux, J.P., 1987, 'Prevalence of enlarged salivary glands in Glossina palpalis, G. pallicera, and G. nigrofusca (Diptera: Glossinidae) from the Vavoua area, Ivory Coast', Journal of Medical Entomology 24(2), 268. http://dx.doi.org/10.1093/ jmedent/24.2.268

Hendrichs, J.P., Kenmore, P., Robinson, A.S. \& Vreysen, M.J.B., 2007, 'Area-wide integrated pest management (AW-IPM): Principles practice and prospects', in M.J.B. Vreysen, A.S. Robinson \& J. Hendrichs (eds.), Area-wide control of insect pests: From research to field implementation, pp. 3-33, Springer, Dordrecht.

Jura, W.G.Z.O., Otieno, L.H. \& Chimtawi, M.M.B., 1989, 'Ultrastructural evidence for trans-ovum transmission of the DNA virus of tsetse Glossina pallidipes (Diptera: Glossinidae)', Current Microbiology 18, 1-4. http://dx.doi.org/10.1007/ BF01568821

Kariithi, H.M., Ahmadi, M., Parker, A.G., Franz, G., Ros, V.I.D, Haq, I. et al., 2013 'Prevalence and genetic variation of salivary gland hypertrophy virus in wild populations of the tsetse fly Glossina pallidipes from southern and eastern Africa', Journal of Invertebrate Pathology 112 (Suppl)., S123-S132. http://dx.doi. org/10.1016/j.jip.2012.04.016

Leak, S.G.A., 1998, Tsetse biology and ecology: Their role in the epidemiology and control of trypanosomosis, CABI Publishing, Wallingford.

Lietze, V.U., Sims, K.R., Salem, T.Z., Geden, C.J. \& Boucias, D.G., 2009, 'Transmission of MdSGHV among adult house flies, Musca domestica (Diptera: Muscidae), occurs via oral secretions and excreta', Journal of Invertebrate Pathology 101(1), 1-7. http://dx.doi.org/10.1016/j.jip.2009.02.007

Malele, I.I., Manangwa, O., Nyingilili, H.H., Kitwika, W.A, Lyaruu, E.A., Msangi, A.R. et al., 2013, 'Prevalence of SGHV among tsetse species of economic importance in Tanzania and their implication for SIT application', Journal of Invertebrate Pathology 112 Suppl, S133-S137. http://dx.doi.org/10.1016/j.jip.2012.07.018

Mutika, G.N., Marin, C., Parker, A.G., Boucias, D.G., Vreysen, M.J.B. \& Abd-Alla, A.M.M., 2012, 'Impact of salivary gland hypertrophy virus infection on the mating success of male Glossina pallidipes: Consequences for the sterile insect technique', PLoS One 7(8), e42188. http://dx.doi.org/10.1371/journal.pone.0042188

Nigatu, R., Rajan, D.S. \& Bizunesh, B.S., 2011, 'Challenges and opportunities in municipal solid waste management: The case of Addis Ababa city, central Ethiopia', Journal of Human Ecology 33(3), 179-190.

Sang, R.C., Jura, W.G.Z.O, Otieno, L.H. \& Mwangi, R.W., 1998, 'The effects of a DNA virus infection on the reproductive potential of female tsetse flies, Glossina morsitans centralis and Glossina morsitans morsitans (Diptera: Glossinidae) Memórias do Instituto Oswaldo Cruz 93, 861-864. http://dx.doi.org/10.1590/ S0074-02761998000600030

Sang, R.C., Jura, W.G.Z.O., Otieno, L.H., Mwangi, R.W. \& Ogaja, P., 1999, 'The effects of a tsetse DNA virus infection on the functions of the male accessory reproductive gland in the host fly Glossina morsitans centralis (Diptera: Glossinidae)', Current Microbiology 38(6), 349-354. http://dx.doi.org/10.1007/PL00006815

Sasanya, J.J., Abd-Alla, A.M.M., Parker, A.G. \& Cannavana A., 2010, 'Analysis of the antiviral drugs acyclovir and valacyclovir-hydrochloride in tsetse flies (Glossine pallidipes) using LC-MSMS', Journal of Chromatography B 878(26), 2384-2390. http://dx.doi.org/10.1016/j.jchromb.2010.07.008 
Shaw, A.P., Cecchi, G., Wint, G.R., Mattioli, R.C. \& Robinson, T.P., 2014, 'Mapping the economic benefits to livestock keepers from intervening against bovine
trypanosomosis in Eastern Africa', Preventive Veterinary Medicine 113(2), 197trypanosomosis in Eastern Africa', Preventive Veterinary
210. http://dx.doi.org/10.1016/j.prevetmed.2013.10.024

Simarro, P.P., Louis F.J. \& Jannin, J., 2003, 'Sleeping sickness, forgotten illness: What are the consequences in the field?', Médecine Tropicale, Revue du Corps de Sante Colonial 63(3), 231-235.

SPSS, 2007, SPSS Statistics Base 17.0 User's Guide, 17th edn., SPSS Inc, Chicago.

Tesfaye, D., Speybroeck, N., De Deken, R. \& Thys, E., 2012, 'Economic burden of bovine trypanosomosis in three villages of Metekel zone, northwest Ethiopia', Tropical Anima Health and Production 44(4), 873-879. http://dx.doi.org/10.1007/s11250-011-9981-3
Thrusfield, M., 2005, Veterinary epidemiology, 2nd edn, Blackwell Science, London.

Verena, U.L., Abd-Alla, A.M.M., Marc, J.B.V., Christopher, J.G. \& Drion, G.B., 2011 'Salivary gland hypertrophy viruses: A novel group of insect's pathogenic viruses', Annual Review of Entomology 56, 63-80. http://dx.doi.org/10.1146/annurevento-120709-144841

Vreysen, M.J.B., Saleh, K.M., Ali, M.Y., Abdulla, A.M. \& Zhu, Z.R., 2000, 'Glossina austeni (Diptera: Glossinidae) eradicated on the island of Unguja, Zanzibar, using the sterile insect technique', Journal of Economic Entomology 93(1), 123-135. http://dx.doi.org/10.1603/0022-0493-93.1.123

World Health Organization, 2003, Report of the Scientific Working Group meeting on African trypanosomiasis, 04-08 June, 2001, Geneva. 